\title{
Contenido de Flúor de las Aguas Naturales de Chile y Recomendaciones para la Suplementación*
}

\author{
Dres. Sonia Guerrero R. 1 - 3, Patricia Cisternas P. 1 - 3, Sra. Silvia González R 2 - 5, Dr. Ricardo Uauy D. $1-4$
}

Fluoride content of water sujplies in Chile

\begin{abstract}
Dentai caries is more prevalent in areas with low fluoride water content. This study mearured the fluoride concentration of 380 water sourees which supply urban centers in Chile. Fluoride was measured using the potenciometric method. Values fluctuated from $0.00 \mathrm{ppm}$ in several places to 2.2 $\mathrm{ppm}$ in the city of Iquique. The mean value for the two northernmost regions of the country was 0.75 ppm; the others had mean values of $0.2 \mathrm{ppm}$ or less. The fluoride content in natural water sources is less than the minimun recommended to prevent caries. Only $4.2 \%$ of the urban population receives an adequate thuoride intake. Age specific recommendations fot supplementation are given according to water fluoride content.
\end{abstract}

La alta incidencia y prevalencia de caries dentarias de nuestra población, especialmente infantil, ha sido pteocupación permanente de la Salud Pública y de quienes luchan por encontrar medidas profilácticas. Por ser las caries de etiolugia multifactorial, es necesario investigar el problema desde varios puntos de vista, para poder establecer un programa de prevención para el país. Uno de los puntos interesantes a analizar en relación a prevención es el flúor, ya que se ha demostrado que es el elemento traza más importante para reforzar la resistencia del esmalte del diente al ataque de caries $^{1-2} \cdot 17-18 \cdot 19-20-21 \cdot 22-23 \cdot 24 \cdot$ 25.

En estudios epidemiológicos en comunidades en que el agua de bebida contiene flúor, en una proporción aproximada a $1 \mathrm{ppm}$, se ha comprobado una menor incidencia de caries de alrededor de un $50 \% 3-4 \cdot 5 \cdot 6 \cdot 7 \cdot 9 \cdot 9 \cdot 10$.

Para establecer la conveniencia de suplementar con flúor el agua de bebida, es necesario conocer el contenido de flúor de las aguas que abastecen a la población. En nuestro país, no existe un catastro actualizado del contenido de flúor de las aguas

${ }^{1}$ División de Nutcición Humana y Ciencias Médicas. Instituto de Nutrición y Tecnologia de los Alimentos (INT A). U. de Chile.

2 Empresa Metropolitana de Obras Sanitarias.

${ }^{3}$ Cirujano - Dentista

${ }^{4}$ Médico-Cirujano, Doctorado en Bioquímica Nutricional.

${ }^{5}$ Licenciado en Química.

* Proyecto fínanciado por la Junta Nacional de Auxilio Escolar y Becas (JUNAEB). Publicación parte de la tesis para optar al cítulo de Magister en Nutrición Humana de las Dras. Sonia Guerrero y Patricia Cisternas. JNA, U. de Chile. naturales. La información que existe es de 1939 y ¿barca sólo algunas ciudades ${ }^{3-11}$.

La Organización Mundial de la Salud, recomienda la adición de flúor hasta alcanzar una concentración de $1 \mathrm{ppm}$ en aquellos lugares en que las aguas naturales no poseen suficiente flúor ${ }^{3}$. En Chile se comenzó a agregar flúor al agua potable en el año 1953, llegando progresivamente a cubrirse hasta el $80 \%$ de las localidades: El programa, por razones administrativas y económicas, no fue constante y por último se dejó de hacer en todo el país en el año $1976^{12}-13-14-15-16$.

Los objetivos de este estudio, fueron medir la cantidad de flúor en las aguas naturales que abastecen a la población y sugerir recomendaciones de suplementación para la población nacional. La investigación, es parte de un programa cuyo propósito es entregar bases para mejorar el nivel de salud bucal de la publación chilena, a través de suplementación con flúor.

\section{MATERIAL Y METODO}

Se realizó un catastro del contenido de flúor de las fuentes de agua natural que abastecen a la población urbana del país en forma centralizada. Para este estudio, se consideró urbanas a todas las ciudades con un mínimo de 5001 habitantes, un area dotada de planta urbanística, servicios y un límite minimo de 40 viviendas agrupadas. Estos requisitos deben concurrir conjuntamente para ser clasificadas como tal, según criterio del Instituto de Estadistica (INE).

Se tomaron 380 muestras de agua cruda que corresponden al total de las fuentes de agua natural del país que abastecen al sector urbano, con una alicuota de $300 \mathrm{cc}$ por cada fuente.

Las muestras fueron tomadas por químicos del Sub -Departamento Calidad de aguas de SENDOS, 
de acuerdo a la norma NCH 411, del Instituto Nacional de Normalización ${ }^{26}$ en frascos de polietileno de $300 \mathrm{cc}$. cada uno, evitando su contaminación y seffalando su identificación.

Las muestras fueron tomadas en el transcurso de un año y como la concentración de fluoruro en el agua natural puede varjar por cambios estacionales y la temperatura del agua, se comparó las muestras en estudio con otras tomadas por SENDOS y Ministerio de Salud en diferentes épocas del año, sin encontrar diferencias significativas entre las diferentes muestras.

Para el análisis del contenido de fluoruros en el agua cruda, se usó el método con electrodo específico para el ión. Este método permite determinar concentraciones con una sensibilidad de hasta $0.01 \mathrm{ppm}$ y una precisión dada por una desviación standar de 0.0016 ppm. ${ }^{26 \cdot 27}$.

Una vez obtenidos los valores de concentración de flúor de las fuentes de abastecimiento, se listaron todas las ciudades de Chile. En cada ciudad se anotó los valores de flúor encontrados y la población estimada por el INE a Diciembre de 1980. Cuando existía más de una fuente de abastecimiento y la diferencia de los valores era menor de $0.1 \mathrm{ppm}$ se procedió a calcular el promedio aritmético. Cuando la diferencia de valores era mayor de $0.1 \mathrm{ppm}$ se procedió a calcular la cantidad de flujo de agua de cada una de las fuentes, traduciéndolo a porcentaje estimativo del aporte, computándose un promedio ajustado. Para la Región Metropolitana se reportan los valores por comuna, por no ser posible determinar en forma exacta la correspondencia entre la fuente y la población abastecida.

\section{RESULTADOS Y DISCUSION}

La Tabla 1, lista el contenido de flúor y población para aquellas ciudades con más de 5001 habitantes.

Se puede apreciar, que en la ciudad de Arica hay $0.75 \mathrm{ppm}$ de flúor en el agua de bebida de la población y que la ciudad de Iquique es la única de Chile que sobrepasa en forma importante la reco. mendación de 1 ppm.

La región comprendida entre las ciudades de Tocopilla y Coquimbo, cuenta con una cantidad recomendable de flúor en el agua de bebida, de Coquimbo al Sur el contenido de flúor en las aguas naturales es escasísimo, siendo prácticamente cero en las regiones del Sur.

En la Figura 1, se muestra la distribución nacional del contenido de flúor por provincia, en categoŕas de 0.00 a $0.25 ; 0.25$ a 0.75 y más de 0.75 ppm.
Tabla 1.

Concentración de Flúor en las aguas naturales para las ciudades con más de 5001 habitantes

\begin{tabular}{|c|c|c|c|}
\hline CIUDAD & Fláor ppm & CIUDAD & Flúor ppm \\
\hline Arica & 0.75 & Pẹtanllor & 0.10 \\
\hline lquique & 2.20 & Talagante & 0.10 \\
\hline Tocopilla & 0.50 & E Monte & 0.16 \\
\hline Chuquicamata & 0.30 & Medipila & 0.21 \\
\hline María Elena & v. 50 & Buin & 0.06 \\
\hline Calamin a & 0.50 & Isia de Maipo & 0.35 \\
\hline Pedro de Valdivla & 0.50 & San Francike de Mostazal & 0.00 \\
\hline Antofagasta & 0.32 & Graneros & 0.12 \\
\hline Trlal & 0.70 & Sewell & 0.20 \\
\hline Chañaral & 1.10 & Rancagua & 0.20 \\
\hline Pucblo Hundido & 1.00 & Machalí & 0.06 \\
\hline Potjerillos & 1.00 & Reno & 0,00 \\
\hline Copiapo & 1.15 & Santa Cruz & 0.13 \\
\hline Vallenar & 1.00 & San Ferrando & 0.00 \\
\hline La Serena & 0.52 & Chimbarongo & 0.00 \\
\hline Coquimbo & 0.52 & Curicó & 007 \\
\hline Vicuna & 0.35 & Molina & 0.00 \\
\hline Andacollo & 0.15 & Constitucion & 0.00 \\
\hline Ovalle & 0.19 & Talca & 0.08 \\
\hline [llopel & 0.00 & San Javier & 0.12 \\
\hline Cabildo & 0.15 & Linares & 0.00 \\
\hline La Ligua & 0.15 & Caequenes & 0.00 \\
\hline E Melón & 0.10 & Parral & 0,00 \\
\hline Quinteto & 0.00 & San Corlos & 0.00 \\
\hline San Felipe & 0.00 & Chillan & 0.00 \\
\hline La Calora & 0.10 & Coclemu & 0.10 \\
\hline Los Andes & 0.05 & Talcahuane & 0.00 \\
\hline Uay , Llay & 0.08 & San Ped to & 0.00 \\
\hline Quillota & 0.08 & Tomé & 0.00 \\
\hline Viß̄a del Mar & 0.00 & Bulnes & 0.00 \\
\hline Valparajso & 0.00 & Penco & 0.00 \\
\hline Vjlla Alemana & 0.00 & Concepción & 0.00 \\
\hline Limache & 0.00 & Chiguayante & 0.00 \\
\hline E Belloto & 0.00 & Lirujuén & 0.00 \\
\hline Quilpué & 0.00 & Sch wager & 0.00 \\
\hline Til - Til & 0.20 & Cosanc] & 0.00 \\
\hline Casablancs & 0.26 & Lota & 0.00 \\
\hline Colirs & 0.12 & Yumbei & 0.00 \\
\hline Curacavi & 0.15 & Atauco & 0.20 \\
\hline San Antonio & 0.19 & Panguguldi & 0.10 \\
\hline Lata & 0.00 & Villarrica & 0.47 \\
\hline Las Angeles & 0.15 & Valdtria & 0.10 \\
\hline Nacirniento & 0.15 & Paillaco & 0.10 \\
\hline Curanilahue & 0.10 & La Uniór & 0.15 \\
\hline Lebu & 0.20 & $R$ io Bueno & 0.10 \\
\hline Cañete & 0.30 & Lanco & 0.10 \\
\hline Mulchén & 0.20 & Osorno & 0.08 \\
\hline Angol & 0.00 & Río Negeo & 0.00 \\
\hline Collipull & 0.00 & Purranque & 0.18 \\
\hline Traiguên & 0.00 & Llanyuíhuc & 0.20 \\
\hline Victoris & 0.07 & Puerto Varas & 0.00 \\
\hline Curacautin & 0.00 & Puerio Montr & 0.02 \\
\hline Lavtaro & 0.27 & Ancud & 0.00 \\
\hline Carahue & 0.00 & Castro & 0.00 \\
\hline Nuera Imperial & 0.00 & Puerto Aysín & 0.00 \\
\hline Temuco & 0.36 & Coihaique & 0.07 \\
\hline Pitrufquén & $0.2 \mathrm{l}$ & Puerto Natales & 0.20 \\
\hline Lencoche & 0.00 & Punta Auenas & 0.20 \\
\hline
\end{tabular}

REGION METROPOLITANA

\begin{tabular}{|c|c|c|}
\hline COMUNAS & Flíor ppm. & COMUNAS \\
\hline $\begin{array}{l}\text { Curacavi } \\
\text { Tl - Til } \\
\text { Colina } \\
\text { Conchalí } \\
\text { Quilicura } \\
\text { Renca } \\
\text { Santiago } \\
\text { Quints Normal } \\
\text { Pudahuel } \\
\text { San Jasí de Mejpo } \\
\text { La Flarida }\end{array}$ & $\begin{array}{l}0.13^{*} \\
0.20 \\
0.12 \\
0.04^{*} \\
0.15 \\
0.03^{*} \\
0.19 \\
0.08 \\
0.04^{*} \\
0.00 \\
0.00\end{array}$ & $\begin{array}{l}\text { La Cistana } \\
\text { San Migurel } \\
\text { Buin } \\
\text { Las Condos } \\
\text { La Reina } \\
\text { Lsle de Maipo } \\
\text { Malleco } \\
\text { Peniaflor } \\
\text { Talagante } \\
\text { El Monte } \\
\text { Padre Hurtado } \\
\text { Meljpilla }\end{array}$ \\
\hline
\end{tabular}

Flior ppm
0.1
0.04
0.06
0.10
0.20
0.35
0.10
0.10
0.10
0.16
0.00
0.21

* Promedio de las diferentes fuentes. 


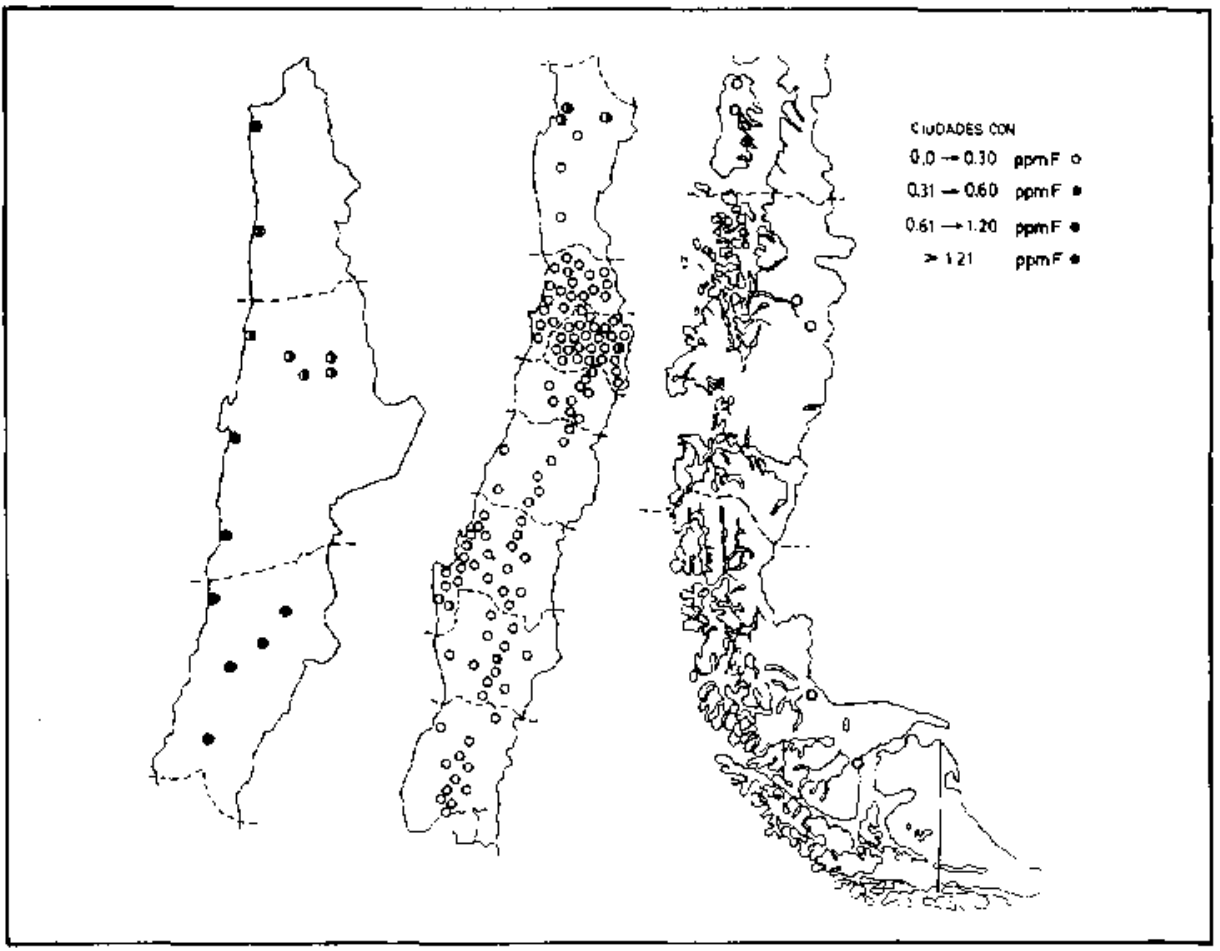

Figura 1.

Cancentración de Flúor en aguas naturales de Chile. En ciudades con más de 5001 habitantes 1981

En la Tabla 2, se puede observar que sólo e] $4,2 \%$ de la población urbana del país recibe flúor de modo natural en cantidad recomendable y que el $95,78 \%$ de la población debería ser suplementado con flúor.

Tabla 2.

Proporción de la población abastecida según concentración de $F$ en las aguas.

Flúor ppm

$$
>0.75
$$

$0.30-0.75$

$0.00-0.30$

Es importante, considerar que 8.346.838 de los 11.198.789 de habitantes estimados como la población de Chile a Diciembre 1980, reciben abastecimiento centralizado de agua potable a través de las fuentes estudiadas, to que representa un $74,5 \%$ de la publación total de Chile. Otras fuentes menores no encuestadas se encuentran preferentemente en las zonas centro y sur, donde por semejanza gevlógica podemos suponer bajos contenidos de flúor.
Mientras no se establezcan programas de suplementación, es fundamental entregar un aporte apropiado de flúor a través de otros vehículos, como gotas o tabletas. Sobre la base de lo anterior, proponemos recomendaciones para suplementación que se resumen en la Tabla 3 y varían según los requerimientos por grupo etario e ingesta habitual de agua. Se ha observado que el contenido de flúor en la leche materna es bajo, independiente del contenido de flúor del agua, por lo tanto, los niños que reciben sólo leche materna, deben recibir suplementación.

Tabla 3.

Recomendación para suplementación con Flúor (mg/dia) según edad y contenido de Flúor del agua

EDAD Concentración Flúor en agua natural ppm $\begin{array}{lllllll}\text { (äios) } \quad 0.2 & 0.2 \ldots & 0.5 & 0.5 & -0.8 & 0.8\end{array}$

$\begin{array}{lllll}<1 & 0.25 & 0^{*} & 0^{*} & 0^{*}\end{array}$

$\begin{array}{lllll}1-3 & 0.50 & 0.25 & 0 & 0\end{array}$

$\begin{array}{lllll}3-6 & 0.75 & 0.50 & 0.25 & 0\end{array}$

$\begin{array}{lllll}>6 & 1.00 & 0.75 & 0.50 & 0\end{array}$

* Excepto para los que reciben alimentación natural exclusiva, que debe ser suplementada con $0.25 \mathrm{mg} / \mathrm{dia}$. 
Considerando los resultados obtenidos, creemos necesario que los profesionales responsables de la salud, establezcan medidas para la suplementación con flúor. En una publicación anterior revisamos el papel del flúor en la prevención de caries, sugiriendo que la suplementación se efectủe desde el nacimiento hasta los 8 años, cuando cesa la actividad amelogénica; después de esta edad el efecto es menor y se obtiene solamente por intercambio iónico en las capas superficiales del esmalte ${ }^{2-9}$. Tal efecto, es el esperado con el uso de pastas dentifricas con flúor, aplicaciones tópicas o enjuagatorios. Estudios realizados en países desarrollados han demostrado el beneficio del flúor local para reducir la prevalencia de caries dentarias dentro de programas integrados con higiene oral $6 \cdot 7 \cdot 10$ $17 \cdot 20$.

A pesar de los esfuerzos que se están realizando para mejorar los hábitos de higiene oral de nuestra población, mediante cepillado sistemático, gran parte de los chilenos no cumplen con estas normas, por lo tanto, es posible esperar que el efecto del flúor tópico alcanzará sólo a una pequeña parte de la población. Los niños y los grupos de bajos ingresos recibirán en mejor forma los beneficios del flúor si este se agrega al agua de bebida o a la dieta, diariamente, en forma de gotas o tabletas.

Por la gran variabilidad del contenido de flúor en las aguas naturales, creemos muy poco posible lograr una ingesta óptima a través del entiquecimiento de alimentos de uso común. Como se ha comprobado en diferentes partes del mundo, creemos que la mejor opción es la fluoración del agua. Tal medida ha resistido todo tipo de crítica, pués no existen efectos tóxicos o teratogénicos atribuibles al flúor si este se emplea en las concentraciones recomendadas y la fluorosis sólo 'se observa cuando el contenido de flúor del agua es mayor que 2 ppm. La fluoración del agua, bien efectuada, consigue concentraciones estables y no produce fluorosis. A pesar de que gran parte del agua fluorada se destina al consumo humano, el costo es significativamente menor que los beneficios económicos obtenidos: menor demanda de atención curativa, menor ausentismo, mayor rendimiento escolar y laboral.

\section{RESUMEN}

Diferentes investigadores han encontrado que la prevalencia de caries dentarias es mayor en las zonas donde el agua no contiene flúor.

Este trabajo, se propuso medir la concentración de fluoruros en las aguas naturales chilenas; calcular la proporción de la población nacional que recibe flúor natural y sugerir recomendaciones para suplementar a su dieta dicho elemento. Para ello, se realizó un catastro del contenido de flúor de las fuentes naturales de agua que en forma centralizada abastecen a la población del pais. Se tomaron 380 muestras de $300 \mathrm{cc}$ cada una, evitando su contaminación. El contenido de fluoruros, se analizó utilizando el método potenciométrico. Los valores fluctúan entre $0.00 \mathrm{ppm}$ hasta $2.2 \mathrm{ppm}$ (Iquique, Canchones), encontrándose un promedio de $0.75 \mathrm{ppm}$ en la I y III Región y un promedio de $0.2 \mathrm{ppm}$ en las regiones restantes.

El contenido de flúor de las aguas naturales, es inferior al mínimo recomendado para la prevención de caries. Sólo un $4.2 \%$ de la muestra alcanza el óptimo recomendado. Aparece fundamental suplementar la dieta con flúor como una de las medidas más importantes para la prevención de caries. Se hacen recomedaciones para suplementación diaria con flúor según edad y contenido de flúor del agua.

\section{AGRADECIMIENTOS}

Los autores agradecen la valiosa colaboración del Sr. Carlos Morales, Ingeniero Jefe del Sub-Departamento Calidad de Aguas SENDOS, y del Sr. Félix Blu, Quimico Jefe Laboratorio EMOS. A la Srta. Genoveva Escobar por su asistencia de secretaria en la preparación de este manuscrito.

\section{REFERENCIAS}

1 Meier, F.: Fluoración del agua potable, OPS/OMS. Publicación No 203, Washington, D.C. 1971

2 Guemero, S., Cisternas, $P, y$ Uouy, R.: Rol del flúor en la prevención de caries dentarias. Rev. Méd. Chile 109 : 55,1981

3 OMS. Fluoruros y Salud. Monografía No 59, Ginebra, Suiza 1972.

4 Meliberg, J.R.: Effect of cariogenic shallenge on fluoride uptake by enamel of rats receiving fluoridate trinking water. Caries Research 12: 137, 1978.

4* Mellterg, J.R.: Enamel fluoride and its anticaries effect. Journal Prev. Dent. 4: 8, 1977.

3 Bojanini, J. y cols.: Siet años de fluoración en Medellín. Rev. Ped. Odont. 24: 31, 1977.

6 Gultow, H,I.: Die lokale anwendung der Aminfluoride auf der Schmelz oberflache als kariesprophylaktish wirksame Massnahme. Deutsche Zahnarztliche Zeitschrift. 23: 898, 1968.

${ }^{7}$ The National Board of Health and Welfare in Sweden Dentol Health in Sweden. Document of Stockhoim Country Council. Dental Public Health. Department of Prevention Dentistry. Stockholm, 1973.

8 Department of Health, Education and Welfare. Documents of fluoride and fluoridation. Public Health Service, Atlanta Georgia Bethesda, Maryland, 1976-1977. 
${ }^{9}$ De ha Fuente $R$ ios $M$. et al.: 11 años de fluoración en Chile, experiencia piloto Curicó - San Fernando 1953--1964. Odont. Chilena 24: 37, 1977.

10 Osvald. O.: The Swedish Public Dental Health Service its development and actual status. Head, Dental Division, National Board of Health Stockholm, 1969.

11 Otte, E.: El contenido de flúor en las aguas potables del país. Rev, Explot. Serv. Agua Potable de Chile 3: 16-17, 1940

12 Latorre. C.: Comunicación Personal.

13 Otre, E.: Comunicación Personal.

14 Unda, F.: Comunicación Personal.

15 Unda, F.: Ingeniería Sanitarik aplicada a Saneamiento y Salud Pública. Unjón típográfica EA. Hispano Americana (UTEMA) 1969. México.

16 Tijmes, B.: Programa Nacional de Fluoración del agua potabie en Chile. Documento Ministerio de Salud Pública. Sery. Nac. de Salud. Sub--Depto. Protección Salud Sección Higiene Ambiental. Santiago de Chile, 1976.

17 Gulzow, $H-J$, und Jellinghous, $M .:$ Kariesprophylaxe durch lokale Application von Fluorid als Losung, Lack oder Gel? Deutsche Zahnarztliche Zeitsche Zeitschrift 28(5): 592-597, 1973 .

18 O.P.S.: Fluoración de la sal. Organización Panameticana de la Salud. Washington, 1976 (Publ. Científ. 335).

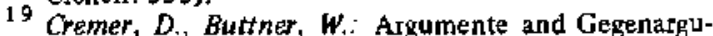
ments Zur Fluoridierung des Trinkwassers. Das offentliche Gesundheitswe gen 33: 166, 1971.

20 Muhlemann, H,R.: Dle Karjesprophylaktische Wirkung der Aminfluoride. Quintessenz 18: 2, 1967.

21 Bramstedt, $F$. und Bandilla, $I$ : Uber den Einfluss organischer Fluorverbindungen auf Saurebildung und Polysaccharidsynthese von Plaques...Streptokken D.Z.Z. 21: $1390,1966$.

22 Arnaudow, M.: Ein Beísfield der Natur. Das offentleche Gesundheitswesen 33: 157, 1971 .

23 Ahrens, G.: Cariesprophylaxe in der Padiatric monatsschrift für kinderheijkunde, $120: 489,1972$.

24 Ophaug, R.H. et al: Estimated fluoride intake of 6 month-old infants in four dietary regions of the United States. The Am. J. of Clin. Nutr. 33: 324, 1980.

25 Singer, $L$, et ol: Fluoride intake of young adults in the United States. The Am. J. of Clin. Nutr. 33: 328, 1980 .

${ }^{26}$ Otiver, R.T. ind Clayton, A.G.: Direct determination of fluoride in micellaneus fluoride materials with the Orion fluoride electrode. Anal. Chim. Acta 51: 409, 1970.

27 APHA - AWWA-WPCF. Standard methods for the examination of water and Wasterwater. 14th. Edition. 1975.

28 Instituto Nacionol de Normalización Inditecnor 62-2ch. Agua para fines industriales: muestras. $\mathrm{NCH} 411,1963$. 